\title{
Operational characteristics of an innovative device dedicated for the hydrodynamic disintegration of sewage sludge
}

\author{
Monika Zubrowska-Sudol ${ }^{1, *}$, Agnieszka Garlicka ${ }^{1}$, Justyna Walczak $^{1}$, \\ Katarzyna Sytek-Szmeichel ${ }^{1}$, Aleksandra Mikołajczak ${ }^{2}$, Michał Stępień2 ${ }^{2}$ Piotr Krawczyk ${ }^{2}$, \\ Katarzyna Umiejewska ${ }^{1}$, and Marcin Wołowicz $^{2}$ \\ ${ }^{1}$ Warsaw University Of Technology, Faculty of Building Services, Hydro and Environmental \\ Engineering, ul. Nowowiejska 20, 00-653 Warsaw, Poland \\ ${ }^{2}$ Warsaw University Of Technology, Faculty of Power and Aeronautical Engineering, \\ ul. Nowowiejska 24, 00-655 Warsaw, Poland
}

\begin{abstract}
This paper presents the results of the first stage of the project, aimed at the assessment of the applicability of the new apparatus for disintegration of excess waste activated sludge. It was documented that the analysed device allows for disintegration of sewage sludge with an efficiency comparable to that obtained in other devices dedicated for mechanical disintegration of sewage sludge described in the literature. The disintegration process at energy density in a range of 35-210 kJ/l resulted in the release from activated sludge flocs of $219-515 \mathrm{mg} \mathrm{SCOD} / \mathrm{l}$ for $35 \mathrm{~kJ} / \mathrm{l}$ to $2138-4884 \mathrm{mg} \mathrm{SCOD} / \mathrm{l}$ for $210 \mathrm{~kJ} / \mathrm{l}$.
\end{abstract}

\section{Introduction}

Pursuant to the hierarchy of waste management options adopted in the Polish National Waste Management Plan [1], in the case of municipal sewage sludge (MSS), "production of MSS should be particularly prevented, for example through subjecting sludge to processing such as disintegration, deep stabilisation, hygienisation, and dewatering, or activities aimed at the loss of its waste status so that MSS can be used as organic fertiliser". The disintegration process mentioned in the cited legal act is usually applied for processing excess waste activated sludge (WAS). In this process, organic and mineral compounds on the surface and inside activated sludge flocs are released to the liquid. Disintegration has been successfully applied to treat WAS in order to intensify its anaerobic digestion [2, 3]. Its benefits include smaller digested sludge mass, reduced digestion time, increased biogas yields, and increased reduction of volatile solids mass. Disintegration of WAS can also be used as a method of production of easily biodegradable organic substrates in order to improve the efficiency of biological nutrient removal form sewage [4].

\footnotetext{
*Corresponding author: monika.sudol@pw.edu.pl
} 
At the Department of Water Supply and Wastewater Management of the Warsaw University of Technology experiments on hydrodynamic disintegration of WAS have been conducted for 10 years. The choice of the disintegration method was determined by the potential to minimise costs of obtaining organic compounds in comparison to other disintegration methods, which is an important factor allowing for the implementation of the investigated solution. Results obtained in the long-term research showed that with properly selected parameters, the process of hydrodynamic disintegration of WAS permits an increase in the efficiency of anaerobic digestion [5], as well as obtaining organic compounds for the improvement of the efficiency of removal of nutrient from sewage $[6,7]$. Considering the current trends in sewage sludge management, the process is also becoming attractive as a pretreatment method for phosphorus and nitrogen recovery. Such broad technological applicability encouraged the search for new construction designs of hydrodynamic disintegration units. For the developed device to be competitive towards solutions currently offered in the market, particular emphasis was put on limiting electricity consumption and reliability of operation. This paper presents the results of the first stage of the project, aimed at the assessment of the applicability of the new apparatus for disintegration of excess waste activated sludge. It involved monitoring of the effects of hydrodynamic disintegration of WAS, conducted with different energy input (defined as energy density $\left(\varepsilon_{\mathrm{L}}\right.$ ) in $\mathrm{kJ} / \mathrm{l}$ and specific energy $\left(\varepsilon_{S}\right)$ in $\left.\mathrm{kJ} / \mathrm{kg} \mathrm{TS}\right)$, on the process of organic compound release from activated sludge flocs and on the activity of biomass.

\section{Material and methods}

\subsection{Characteristics of the disintegration apparatus - principle of operation}

A schematic diagram of the innovative device dedicated for hydrodynamic disintegration of biological substrates via the cavitation process is presented in Fig.1. The device consists of a rotor driven by an electric motor, placed in a cylindrical tank with a total volume of approx. 13 1. The cavitation phenomenon occurs in two zones. The first one contains axial rotor channels. During the device operation, the rotor has significant torque, and the in-channel liquid velocity is very high. The rotational speed causes a pressure drop, resulting in cavitation. The second zone is the area where the substrate leaves the rotor and strikes the stiffening ribs with high linear velocity. The working space consists of two concentric, thinwalled cylinders connected by the ribs. The substrate leaves the rotor and is superseded by the other part of substance staying between the cylinders. Therefore, the entire substance circulates in the working space and comes across the cavitation area many times during the operation. The circulation intensifies the cavitation process. The chamber is confined from the bottom with a profiled plate, and from the top with a cover. The profiled plate is equipped with a central hole containing the shaft transferring the drive from the electric motor to the device rotor and its sealing. In order to prevent liquid deposits inside the device, and to counteract the motion of substrate in the direction of the rotation axis, an elliptical, slanted channel and conic base were introduced at the bottom of the device, facilitating emptying the chamber. The cover can be opened to load, and then closed during the operation, or adapted to continuous work. In this regime, the cover can be equipped with an inlet pipe and be constantly operated in technological line. The cover is currently sealed with a gasket frame. The rotor is a steel cylinder with circumferential, profiled channels. It is made of material designed for aggressive work environment, and is resistant to corrosion. The rotor cavitation area is closed by two discs. The upper disc is equipped with a mechanism causing the substrate suction into the rotor. The device has a set of auxiliary equipment, e.g. energy consumption measurement system, inverter for the rotation speed change, or a controller 
monitoring other operational parameters. The most important advantages of the device are as follows: simplicity and controllability of the mechanism; compact shape; ease of modification; high fatigue life caused by maximum reduction of live elements; high efficiency.

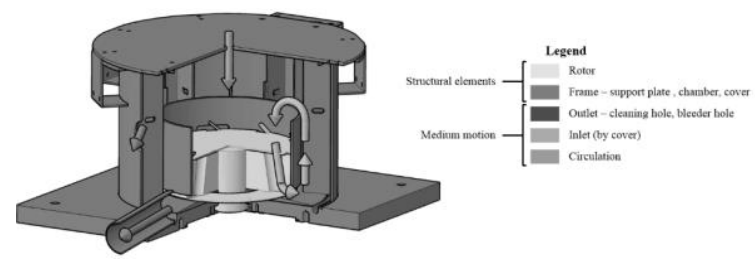

Fig. 1 Schematic diagram of the device.

\subsection{Experimental setup}

In order to assess the efficiency of the disintegration process implemented in the new device, seven disintegration batch tests were performed with the following defined partial objectives: 1) analysis of organic compound release from activated sludge flocs as a function of energy input in the disintegration process, 2) analysis of microbial activity as a function of energy input in the disintegration process. The methodology of the disintegration tests was described in detail in the paper [8]. For each of the disintegration batch tests, a new portion of WAS was delivered from a local biological nutrient removal WWTP in Warsaw (Poland). WAS for the tests was collected directly from the output of the centrifuge. Total solid (TS) and total volatile solid (VTS) concentrations in WAS used in the experiment were 5.19-5.79\% and $3.77-4.33 \%$, respectively. The principle of the disintegration batch test was to measure the physico-chemical indicators of: 1) non-disintegrated sludge and 2) sludge after introducing a selected portion of energy expressed as energy density. In each test, a 10 litre of WAS was subject to the disintegration process at six levels of $\varepsilon_{\mathrm{L}}$, modified in a range 35-210 kJ/l with a $35 \mathrm{~kJ} / \mathrm{l}$ step. The scope of the analysis included: 1) TS and VTS concentrations in sludge before disintegration; 2) temperature in sludge before and after disintegration; 3) characteristics of the liquid phase in the samples before and after disintegration: chemical oxygen demand (SCOD), volatile fatty acids (VFA) and $\mathrm{pH}$; the liquid phases were obtained by $30 \mathrm{~min}$ centrifugation at a speed of $15000 \mathrm{rpm}$, and filtration on filter paper with a pore diameter of $0.45 \mu \mathrm{m}$; 4 ) degree of sludge disintegration ( $\mathrm{DD}_{\mathrm{COD}}$ ) according to Nickel and Neis [9]; 5) total activity degree (ADouR) according to [8]. All chemical analyses were performed in duplicates in accordance with APHA Standard Methods [10].

\section{Results and discussion}

\subsection{Temperature changes in the hydrodynamic disintegration process}

Hydrodynamic cavitation occurs as a result of a decrease in static pressure in the liquid below critical pressure. A decrease in pressure can be caused by a local increase in flow rate or a change in external conditions, e.g. pressure, resulting in the development of areas of discontinuity in the medium, filled with vapour or gas (microbubbles), subject to rapid implosion. During the implosion, the temperature inside the imploding bubble can considerably increase, resulting in the generation of substantial amounts of heat to the surroundings, causing a general increase in temperature in the disintegrated sludge [11]. 
Table 1. Effect of energy density on temperature and disintegration degree.

\begin{tabular}{|c|c|c|c|c|c|c|c|}
\hline & \multicolumn{3}{|c|}{$\mathrm{DD}_{\mathrm{COD}}(\%)$} & \multicolumn{3}{|c|}{ Temperature $\left({ }^{\circ} \mathrm{C}\right)$} \\
\hline & & Min & $\operatorname{Max}$ & Average \pm SD & Min & Max & Average $\pm \mathrm{SD}$ \\
\hline \multicolumn{2}{|c|}{ non-disintegrated WAS } & - & - & - & 11.6 & 24.7 & $19.9 \pm 5.5$ \\
\hline \multirow{6}{*}{$\begin{array}{c}\text { WAS after } \\
\text { disintegration } \\
\text { with energy } \\
\text { input }\end{array}$} & $35 \mathrm{~kJ} / \mathrm{l}$ & 0.7 & 1.8 & $1.1 \pm 0.4$ & 24.0 & 30.8 & $28.4 \pm 2.4$ \\
\hline & $70 \mathrm{~kJ} / \mathrm{l}$ & 1.3 & 3.4 & $2.1 \pm 0.8$ & 29.4 & 36.3 & $33.9 \pm 2.7$ \\
\hline & $105 \mathrm{~kJ} / \mathrm{l}$ & 2.3 & 4.7 & $3.1 \pm 0.9$ & 34.9 & 42.7 & $38.8 \pm 3.1$ \\
\hline & $140 \mathrm{~kJ} / \mathrm{l}$ & 3.0 & 8.0 & $4.7 \pm 1.8$ & 39.6 & 47.7 & $43.2 \pm 3.5$ \\
\hline & $175 \mathrm{~kJ} / \mathrm{l}$ & 4.7 & 13.2 & $7.9 \pm 3.7$ & 42.9 & 54.4 & $47.4 \pm 4.3$ \\
\hline & $210 \mathrm{~kJ} / \mathrm{l}$ & 7.0 & 17.0 & $10.8 \pm 3.9$ & 45.7 & 59.0 & $51.0 \pm 4.9$ \\
\hline
\end{tabular}

Description: Min - minimum value; Max - maximum value; SD - Standard deviation.

In accordance with the results presented in Table 1, an increase in energy density was observed to be accompanied by a gradual increase in temperature from $19.9 \pm 5.5^{\circ} \mathrm{C}$ for non-disintegrated sludge to $51.0 \pm 4.9^{\circ} \mathrm{C}$ for disintegrated sludge at $\varepsilon_{\mathrm{L}}=210 \mathrm{~kJ} / \mathrm{l}$. The observation was the first signal of the occurrence of cavitation in the new, innovative device. Similar changes in temperature were recorded by Huan et al. [12] and Xu et al. [13], respectively for ultrasound disintegration and for combined treatment of ultrasound and ozone. It is worth emphasising that an excessive increase in temperature leads to a reduction of surface tension, facilitating the development of gas bubbles with a high content of vapour inhibiting their breakage [14]. In the cited research, the value of pressure oscillation caused by bursting of cavitation bubbles increased at a temperature of up to $40^{\circ} \mathrm{C}$, and then considerably decreased at a temperature of $69.5^{\circ} \mathrm{C}$. An increase in temperature was accompanied by an increase in vapour density, signalling a requirement of more heat to obtain the same amount of vapour. Heat flow from the liquid to vapour causes a decrease in the temperature of the liquid and a local decrease in evaporation pressure. The development of another cavitation bubble is initiated at lower pressure, and the bubble grows to a smaller size. The aforementioned observations were obtained in experiments on the process of hydrodynamic cavitation for the medium of water [14].

\subsection{Effects of hydrodynamic disintegration on organic compound release}

One of the most frequently applied methods of assessment of disintegration efficiency is the analysis of changes in soluble COD (SCOD), and determination of the related degree of disintegration $\left(\mathrm{DD}_{\mathrm{COD}}\right)$. Because disintegration leads to a transformation of non-soluble organic compounds (constituting building material of extra- and intracellular structures of active sludge flocs) into soluble compounds, the process is expected to result in an increase in the value of SCOD, and the magnitude of the increase provides the basis for the assessment of disintegration efficiency. In all tests conducted for the new device, an increase in energy

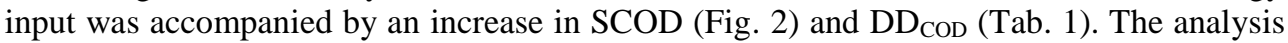
of the relationship between energy input (expressed as energy density and specific energy) and SCOD showed that in each case a specific threshold of energy input occurred above which the intensity of release of organic compounds substantially increased. It is in line with the results our previous studies conducted for two different devices dedicated for hydrodynamic disintegration of sewage sludge $[8,15]$, and with the results of Biradar et al. [16] who also analysed hydrodynamic disintegration. In five out of seven conducted tests, it was $\varepsilon_{\mathrm{L}}$ value of $140 \mathrm{~kJ} / \mathrm{l}$. Considering the TS value for the tests remaining in the range of $5.19-5.79 \%$, it corresponded with $\varepsilon_{\mathrm{S}}$ of $2400-2700 \mathrm{~kJ} / \mathrm{kg} \mathrm{TS}$. 


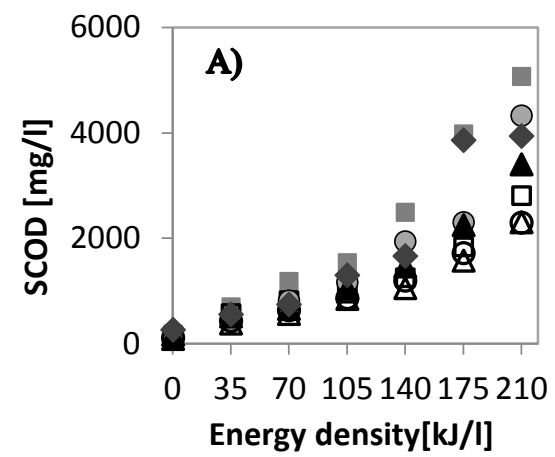

$$
\begin{aligned}
& \text { T1 (TS }=5,22 \%) \\
& \text { O T3 (TS }=5,52 \%) \\
& \triangle \text { T5 (TS }=5,56 \%)
\end{aligned}
$$$$
\text { - T7 (TS = 5,20\%) }
$$

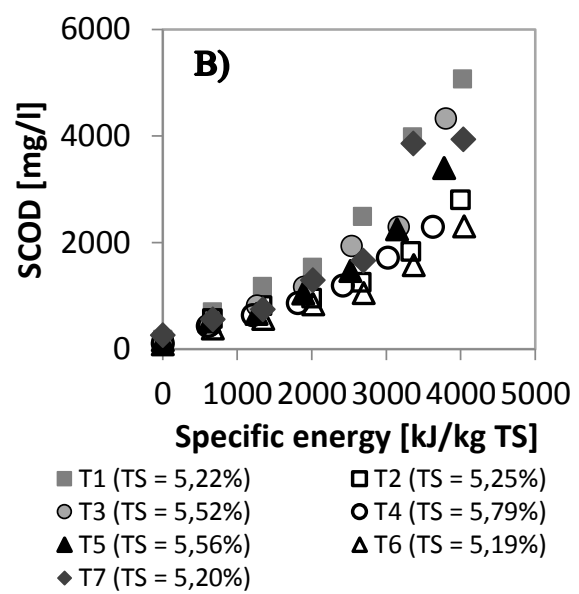

Fig. 2. SCOD vs energy input expressed as energy density (A) and specific energy (B).

In the remaining two tests (T1 and $\mathrm{T} 3$ ), the threshold was determined for $\varepsilon_{\mathrm{L}}$ equal to $105 \mathrm{~kJ} / 1$, corresponding with specific energy of $2010 \mathrm{~kJ} / \mathrm{kg}$ TS for T1 and $1990 \mathrm{~kJ} / \mathrm{kg}$ TS for T3. For example, in test No. 1, below the threshold, the amount of released organic compounds between subsequent identical portions of energy input in the disintegration process $(35 \mathrm{~kJ} / 1$, corresponding to $670 \mathrm{~kJ} / \mathrm{kg} \mathrm{TS})$ remained in a range $360-515 \mathrm{mg} \mathrm{SCOD} / \mathrm{l}$, and above it increased to 950-1490 mg SCOD/l. Data presented in the Fig. 2 also show that both below and above the so-called threshold value of energy input, the relationship between energy input and SCOD was close to positive linearity, whereas the function describing the distribution of points above the threshold value was characterised by a higher directional coefficient. It is worth mentioning that the linear relationship between SCOD and $\varepsilon_{\mathrm{S}}$ remaining in a range of 200-3000 kJ/kg TS, analogical to that of $\varepsilon_{\mathrm{S}}$ below the threshold value in our study, was recorded by Lippert et al. [17]; (the cited authors analysed ultrasound disintegration). As expected, the new device also allowed for the acidification process, as suggested by an increase in concentration of VFA in the samples after the disintegration process in comparison to the sample before the process (Fig. 3). It is worth emphasising that in spite of differences between VFA concentration values that occurred for the same $\varepsilon_{\mathrm{L}}$ levels in particular disintegration tests, a linear correlation was found between the concentration of

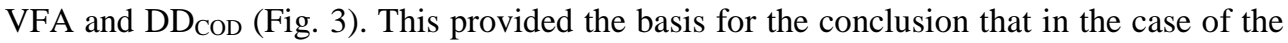
analysed range of energy input in the disintegration process, the primary factor determining the course of acidification was the degree of disintegration. During our long-term research on the process of disintegration of sewage sludge it was observed that even when it is conducted in the same device, with the same energy input (expressed as energy density or specific energy), for sludge from the same sewage treatment plant and characterised by similar dry mass content, the values of indicators providing the basis for the determination of the efficiency of the disintegration process, obtained for different parts of sludge, remain within quite broad ranges [among others 8,18 ]. It is also documented by results presented in this paper (Fig. 2 and Fig. 3). The phenomenon can be explained by the non-uniform structure of WAS, depending on a high number of factors, on the one hand related to biological sewage treatment, and on the other hand to the process of sludge thickening. The propagation and activity of forces causing disintegration can be determined by sludge properties such as: size and porosity of activated sludge flocs, durability of bonds between particular structures of activated sludge flocs, amount and structure of extracellular substances, or type of microorganisms inhabiting the flocs. 

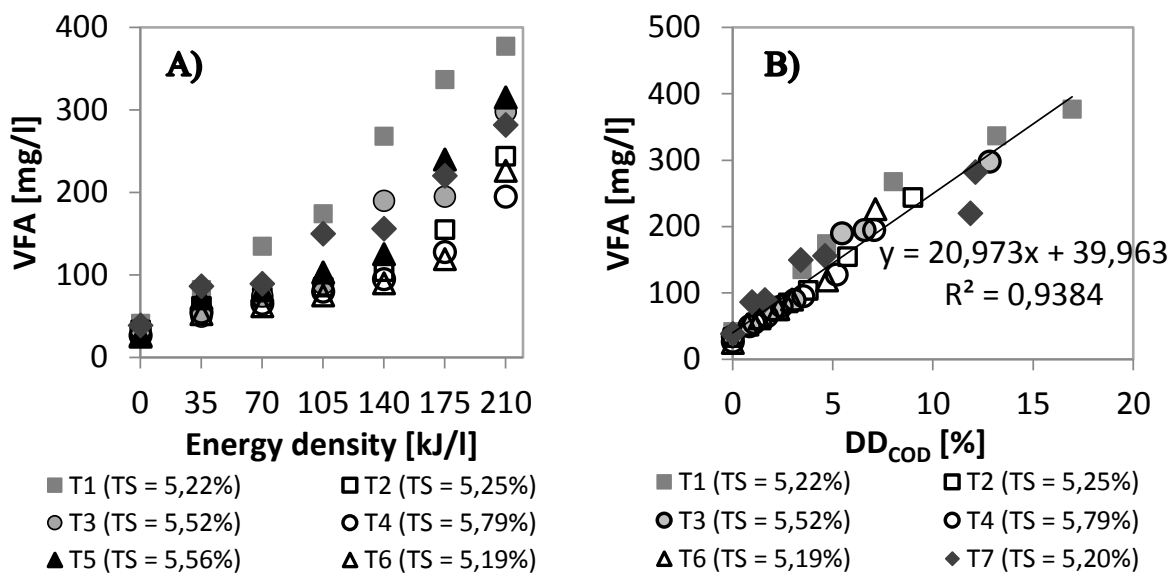

Fig. 3. VFA concentration vs. energy density (A) and degree of disintegration (B).

Considering the above, in order to unambiguously compare the efficiency of the disintegration process implemented in different devices, the experiment should be conducted for the same portion of WAS. The comparison of results obtained in independent experiments only provides a general overview of the efficiency of disintegration obtained in the analysed device. It constitutes an important element of the assessment of the operational characteristics of the new device, however, particularly in reference to the relationships between energy inputs required for the disintegration process and the process efficiency. The comparison of the effects of disintegration obtained in the new device with those obtained in our earlier research on hydrodynamic disintegration $[8,18]$, conducted in a device described in detail in the paper [8], shows their comparability. In the cited studies, SCOD in disintegrated sludge at $\varepsilon_{\mathrm{L}}$ in a range of 70-210 kJ/l varied from 435 to $3396 \mathrm{mg} / \mathrm{l}$ (sludge used for the experiments was collected from the same sewage treatment plant as that in this study) [8], and from 380 to $6515 \mathrm{mg} / \mathrm{l}$ (sludge used for the experiments was collected from a sewage treatment plant other than the one in this study) [18]. The comparison of the disintegration efficiency obtained in the analysed device with the results of other research groups dealing with hydrodynamic disintegration of WAS [16, 19-21] shows that the new device permitted release from WAS of at least comparable, or even higher amounts of organic compounds. For example, Mancuso et al. [19] with the application of higher $\varepsilon_{\mathrm{S}}$ from 3276 to $12780 \mathrm{~kJ} / \mathrm{kgTS}$ obtained considerably lower SCOD (from 692 to $2436 \mathrm{mg} / \mathrm{l}$ ) than those obtained in this study. Biradar et. al. [16], conducting the process at $\varepsilon_{S}$ of 2000-8000 kJ/kg TS obtained SCOD in a range from 2213 to $2332 \mathrm{mg} / \mathrm{l}$ which for the upper threshold of $\varepsilon_{S}$ was a value considerably lower than those obtained for the analysed device. Results obtained for the new hydrodynamic disintegrator were also compared with results described in the literature for the ultrasound method [17, 22, 23]. The analysis showed that the new device is characterised by higher efficiency of SCOD release at considerably lower $\varepsilon_{\mathrm{S}}$ inputs in comparison to ultrasound disintegration described by Lizama et al. [23]. It permitted obtaining effects comparable to those described by Lippert et al. [17] for sludge from WWTP Munich East (Germany) (Es: 200-3000 kJ/kgTS; SCOD: 500-2 $700 \mathrm{mg} / \mathrm{l}$ ) and by Koch et al. [22] ( $(\mathrm{s}=3440 \mathrm{~kJ} / \mathrm{kg} \mathrm{TS}, \mathrm{SCOD}=3195 \mathrm{mg} / \mathrm{l})$. 


\subsection{Effects of hydrodynamic disintegration on the activity of biomass}
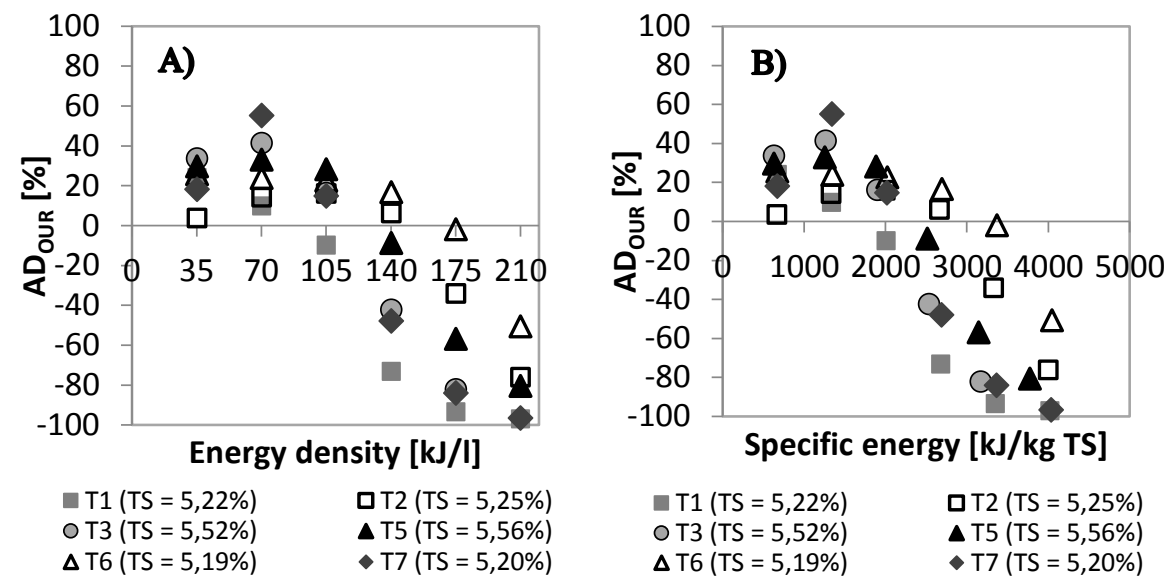

Fig. 4. Total activity degree vs energy input express as energy density (A) and specific energy (B).

The effect of disintegration can also be assessed through the determination of the oxygen uptake rate (OUR) for a sample of activated sludge before and after the disintegration process. If OUR is higher in the sample after disintegration, positive values of the total activity degree ( $\left.\mathrm{AD}_{\mathrm{OUR}}\right)$ are obtained, and the phenomenon is interpreted as an increase in biomass activity. A lower oxygen uptake rate in the sample after disintegration, and therefore a negative value of $\mathrm{AD}_{\text {our }}$, points to the deactivation of the biomass inhabiting activated sludge flocs [8]. Next to the assessment of the effect of disintegration on the activity of aerobic microorganisms, OUR test results are also helpful in the interpretation of results illustrating release of organic compounds from activated sludge flocs. When ADour adopt values higher or equal to 0 , it is highly probable that the released organic compounds originate only from extracellular structures (no breakage of cellular walls occurs). When values of $\mathrm{AD}_{\text {our }}$ are lower than 0 , the released organic compounds can originate from the decomposition of both extra- and intracellular structures. The relationship between energy input and $\mathrm{AD}_{\text {our }}$ is displayed in Fig. 4 The comparison of the relationships with the results presented in Fig. 4 shows that deactivation of the biomass (negative ADour values) occurred when the energy input in the disintegration process was at least equal to the threshold value described in the previous chapter. The observations suggest that the organic compounds released in the disintegration process conducted until reaching the threshold value originated from the decomposition of extracellular substances. After its exceedance, destruction of intracellular substances had an increasing effect on the obtained SCOD values. It is also noteworthy that depending on the energy density, the course of $\mathrm{AD}_{\mathrm{OuR}}$ values determined for the new device is analogical to that determined in earlier studies of the authors [8].

\section{Conclusion}

In reference to the main study objective, namely the assessment of the applicability of the new apparatus for disintegration of excess waste activated sludge, it can be concluded that the analysed device allows for disintegration of sewage sludge with an efficiency comparable to that obtained in other devices dedicated for mechanical disintegration of sewage sludge described in the literature. The disintegration process at $\varepsilon_{\mathrm{L}}$ in a range of $35-210 \mathrm{~kJ} / \mathrm{l}$ resulted in the release from activated sludge flocs of $219-515 \mathrm{mg} \mathrm{SCOD} / \mathrm{l}$ for $35 \mathrm{~kJ} / \mathrm{l}$ to 
2138-4884 mg SCOD/1 for $210 \mathrm{~kJ} / \mathrm{l}$. The simultaneously conducted OUR tests showed that if the disintegration process was conducted at $\varepsilon_{\mathrm{L}}$ lower than $105 \mathrm{~kJ} / \mathrm{l}$, the released organic compounds originated from the destruction of extracellular substances. At higher energy densities, an increasing role was played by the process of destruction of intracellular substances. Further works on the flow system of the disintegrator are planned, aimed at the modification of the rotor to obtain higher disintegration efficiencies at lower energy input. The modified device will also be adjusted to processing substrates used in agricultural biogas plants (such as silage, fruit and vegetable waste, sugar beetroot pulp, etc.).

The study was implemented in the scope of a research project entitled "Development of a technology for preparation substrates used in methane co-fermentation by disintegration methods" (DEZMETAN) (No. POIR.04.01.02-00-0022/17), financed in the scope of Measure 4.1 of the Operational Programme Smart Growth 2014-2020 co-financed from the resources of the European Regional Development Fund.

\section{References}

1. Polish National Waste Management Plan 2022. Polish Monitor dated 11.08.2016 poz. 784. In Polish. Krajowy plan gospodarki odpadami 2022. Monitor Polski z dnia 11.08.2016 poz. 784

2. P. N. Patil, P. R. Gogate, L. Csoka, A. Dregelyi-Kiss, M. Horvath, Ultrason. Sonochem. 30, 79-86 (2016)

3. L. Peng, L. Appels, H. Su, J. Environ. Manage. 213, 271-278 (2018)

4. R. Xu, Y. Fan, Y. Wei, Y. Wang, N. Luo, M. Yang, X. Yuan, R. Yu, J Environ Sci. 48, 59-68 (2016)

5. M. Zubrowska-Sudol, J. Podedworna, K. Sytek-Szmeichel, A. Bisak, P. Krawczyk, A. Garlicka, TSEP 5, 289-295 (2018)

6. M. Zubrowska-Sudol, J. Walczak, Water Res. 76, 10-18 (2015)

7. J. Walczak, M. Zubrowska-Sudol, Water Sci. Technol. 77, 9, 2165-2173 (2018)

8. M. Zubrowska-Sudol, J. Walczak, Water Res. 61, 200--209 (2014)

9. K. Nickle, U. Neis, Ultrason. Sonochem. 14, 450-455 (2007)

10. Standard Methods for the Examination of Water and Wastewater $\left(20^{\text {th }}\right.$ ed. APHA, AWWA \& WEF, Washington, DC, USA, 1998)

11. H. Zhao, P. Zhang, G, Zhang, R. Cheng, J. Environ Sci. 42, 163-167 (2016)

12. L. Huan, J. Yiying, R.B. Mahar, W. Zhiyu, N. Yongfeng, J. Hazard. Mater. 161, 1421- 1426 (2009)

13. G. Xu, S. Chen, J. Shi, S. Wang, G. Zhu, J. Hazard. Mater. 180, 1-3, 340-346 (2010)

14. A. Šarc, T. Stepišnik-Perdih, M. Petkovšek, M. Dular, Ultrason. Sonochem. 34, 51-59 (2017)

15. M. Żubrowska-Sudoł, J. Podedworna, Z. Heidrich, P. Krawczyk, J. Szczygieł, Environ. Engineering 3, 257-261 (2010)

16. P. M. Biradar, S. B. Roy, S. F. D’Souza, A. B. Pandit, Bioresour. Technol. 101, 1787-1791 (2010)

17. T. Lippert, J. Bandelin, A. Musch, J. E. Drewes, K. Koch, Bioresour. Technol. 264, 298-305 (2018)

18. J. Walczak, M. Zubrowska-Sudol, P. Piechna, Clean. 45, 11, 1700487 (2017)

19. G. Mancuso, M. Langone, G.Andreottola, Ultrason. Sonochem. 35, 489-501 (2017) 
20. M. Petkovšek, M. Mlakar, M. Levstek, M. Stražar, B. Širok, M. Dular, Ultrason. Sonochem. 26, 408-414 (2015)

21. K. W. Jung M. J. Hwang, Y. M. Yun, M. J. Cha, K. H. Ahn. Ultrason. Sonochem. 21, 5, 1635-1640 (2014)

22. K. Koch, T. Lippert, N. H. Sabadini, J. E. Drewes, Ultrason. Sonochem. 37, 464-470 (2017)

23. A. C. Lizama, C. C. Figueiras, R. R. Herrera, A. Z. Pedreguera, J. E. R. Espinoza, Int. Biodeterior. Biodegradation. 123, 1-9 (2017) 